\title{
Characterization of an Alcoholic Hepatic Steatosis Model Induced by Ethanol and High-Fat Diet in Rats
}

\author{
Carlos Eduardo Alves de Souza ${ }^{1}$, Aline Maria Stolf ${ }^{1}$, Arturo Alejandro Dreifuss ${ }^{1}$, \\ Francislaine dos Reis Lívero ${ }^{1}$, Liana de Oliveira Gomes ${ }^{1}$, Lyvia Petiz ${ }^{2}$, Olair Beltrame ${ }^{3}$, \\ Rosangela Locatelli Dittrich ${ }^{3}$, José Ederaldo Queiroz Telles ${ }^{4}$, Sílvia Maria Cadena ${ }^{2}$ and \\ Alexandra Acco ${ }^{1 *}$ \\ ${ }^{I}$ Departamento de Farmacologia; Universidade Federal do Paraná; Curitiba - PR - Brasil. ${ }^{2}$ Departamento de \\ Bioquímica e Biologia Molecular; Universidade Federal do Paraná; Curitiba - PR - Brasil. ${ }^{3}$ Departamento de \\ Medicina Veterinária; Universidade Federal do Paraná; Curitiba - PR - Brasil. ${ }^{4}$ Departamento de Patologia \\ Médica; Curitiba - PR - Brasil.
}

\begin{abstract}
Alcoholic liver disease is characterized by a wide spectrum of liver damage, which increases when ethanol is associated with high-fat diets (HFD). This work aimed to establish a model of alcoholic hepatic steatosis (AHS) by using a combination of $10 \%$ ethanol and sunflower seeds as the source of HFD. Male rats received water or $10 \%$ ethanol and regular chow diet and/or HFD, which consisted of sunflower seeds. The food consumption, liquid intake and body weight of the rats were monitored for 30 days. After this period, blood was collected for biochemical evaluation, and liver samples were collected for histological, mitochondrial enzyme activity and oxidative stress analyses. Our results indicated that the combination of $10 \%$ ethanol and HFD induced micro-and macrosteatosis and hepatocyte tumefaction, decreased the levels of reduced glutathione and glutathione S-transferase activity and increased the level of lipoperoxidation and superoxide dismutase activity. The mitochondrial oxidation of NADH and succinate were partially inhibited. Complexes I and II were the main inhibition sites. Hepatic steatosis was successfully induced after 4 weeks of the diet, and the liver function was modified. The combination of 10\% ethanol and sunflower seeds as an HFD produced an inexpensive model to study AHS in rats.
\end{abstract}

Key words: Ethanol, alcoholic hepatic steatosis, high-fat diet, sunflower seeds, mitochondria

\section{INTRODUCTION}

Alcoholic dependency is considered to be a worldwide public health problem, and a direct causal relationship has been observed between alcohol consumption and more than 60 different types of diseases and injuries, including those in the liver (Miranda-Mendez et al. 2010). Alcoholic liver disease (ALD) is characterized by a wide spectrum of liver damage, ranging from liver steatosis (fatty liver) to steatohepatitis and liver fibrosis/cirrhosis. It is believed that the obvious cause for ALD is hepatocyte death induced by alcohol itself (Byun and Jeong 2010; CurryMcCoy 2010). However, the involvement of oxidative injury in ethanol toxicity has emerged from a growing number of reports that showed that alcohol-fed experimental animals, as well as patients with ALD, have increased contents of lipid peroxidation products in the liver and in the blood (Albano 2008).

*Author for correspondence: aleacco@ufpr.br 
Mechanisms implicated in alcohol-induced liver damage involve many biochemical reactions, with different pathways interacting with each other simultaneously. These mechanisms involve enzymes, reactive oxygen species (ROS), endotoxins, cytokines, immune system cells, and genetic predisposition to liver disease ( $\mathrm{Lu}$ and Cederbaum 2008; Miranda-Mendez et al. 2010). The intracellular accumulation of lipids is the most frequent liver lesion in heavy drinkers. The impairment of mitochondrial lipid oxidation has been proposed as one of the mechanisms that is responsible for this fat accumulation (Pessayre and Fromenty 2005; Pessayre and Fromenty 2012). Oxidative stress associated with alcohol toxicity is mainly caused by ROS generated by the mitochondrial respiratory chain, by the enzyme responsible for the ethanol metabolism (CYP2E1) in hepatocytes, and by the NADPH oxidase of Kupffer cells and liver-infiltrating granulocytes. In addition, the oxidation of ethanol through the alcohol dehydrogenase pathway produces acetaldehyde, which is converted to acetate. Both reactions promote an increase of $\mathrm{NADH}$, which, in excess, results in several metabolic disorders, including the inhibition of fatty acid oxidation and tricarboxylic acid cycle (Tilg et al. 2011). Therefore, it increases the hepatic fat accumulation.

For decades, dietary deficiencies were considered the major factor responsible for the development of liver disease in alcoholics (Korourian et al. 1999), because ethanol displaces normal nutrients, causing malnutrition (Liber 2004; Comporti et al. 2010). Moreover, it has been experimentally proved that nutritional deficiencies cause liver damage (Di Pascoli et al. 2004; Rautou et al. 2008; Caballero et al. 2011). Therefore, it was postulated that the combination of these factors cause ALD (Comporti et al. 2010). For instance, it has been reported that a low-carbohydrate diet associated with ethanol induced severe hepatotoxicity with intense steatosis (Korourian et al. 1999). Despite alcoholic fatty liver has long been considered benign, increasing evidence supports the idea that steatosis may contribute to the progression of other hepatic injuries (Powel et al. 2005).

Because the interaction between ethanol and nutrients may contribute significantly to the pathology of an alcoholic liver injury, the aim of this study was to establish and characterize an alcoholic hepatic steatosis (AHS) model induced by ethanol associated with a high-fat diet (HFD). Because specific chows with high fat or low protein contents, both used in combination with ethanol to induce the steatosis model, are expensive, the purpose of this study is to propose a cheaper model of alcoholic steatosis by using sunflower seeds (Helianthus annuus) as the unique source of the HFD. This study focused on the hepatic oxidative stress and the mitochondrial dysfunction induced by the combination of the liquid diet (10\% ethanol) and HFD (sunflower seeds).

\section{MATERIAL AND METHODS}

\section{Animal care, diets, and sample collection}

Male Wistar rats (Rattus norvegicus) weighing $200 \pm 20 \mathrm{~g}$ were used for this study. The experimental study (Fig. 1) was approved by the Institutional Animal Ethics Committee of the Biological Sciences Sector of the Federal University of Paraná (certificate \#584). Briefly, each animal was housed in a single cage with food and water ad libitum (4 weeks) and maintained at room temperature $\left(22^{\circ} \mathrm{C} \pm 2{ }^{\circ} \mathrm{C}\right)$ on a $12 / 12 \mathrm{~h}$ light/dark cycle. After acclimation for 1 week, the animals were separated into 6 groups $(\mathrm{n}=10$ each) according to their liquid and solid diets: (1) water and chow diet (WC), (2) water and high-fat diet with only sunflower seeds (WH), (3) water and chow plus sunflower seeds (WCH), (4) ethanol and chow diet (EtC), (5) ethanol and highfat diet with only sunflower seeds (EtH), and (6) ethanol and chow diet plus high-fat diet with sunflower seeds $(\mathrm{EtCH})$. Food (chow and/or sunflower seeds) consumption, liquid intake (water or ethanol), and body weight were monitored 3 times per week during a 1-month period. The consumption of sunflower seeds was calculated using only the weight of the peeled seed, which was $48 \%$ of the total weight of the seed.

The nutritional values of the regular chow and the sunflower seeds are shown in the Table 1. After 30 days of consumption of the diet, the animals were anesthetized with ketamine $\left(60 \mathrm{mg} \cdot \mathrm{kg}^{-1}\right.$ Vetnil, Louveira, SP, Brazil) and xylazine (7.5 mg. $\mathrm{kg}^{-1}$ - König, Santana de Parnaíba, SP, Brazil) by intraperitoneal injection. Blood from individual rats was collected from the abdominal cava vein to determine the plasma levels of biochemical parameters. The liver was harvested, weighted, 
and quickly snap-frozen in liquid nitrogen and stored at $-80^{\circ} \mathrm{C}$ for hepatic oxidative stress analysis and quantification of lipids. A portion of the liver was fixed in 10\% neutral-buffered formalin for histological analysis. For isolation of liver mitochondria, the animals were euthanized by decapitation.

\section{Hepatic histology}

The liver tissue, which was fixed in $10 \%$ (v/v) neutral-buffered formalin, was further embedded in paraffin at room temperature. Thin sections
(4 mm) from paraffin blocks were processed for histology, and the tissue was stained with hematoxylin-eosin according to the routine technique applied at the Department of Medical Pathology of Federal University of Paraná. Another liver tissue fraction was embedded in Tissue-Tek Optimal Cutting Temperature (O.C.T.) compound (Sakura, Torrance, CA, USA), processed in a cryostat, and stained with Sudan Black, which is specific stain for lipids. All slides were then analyzed by light microscopy (Leica DM 2500, Wetzlar, Germany).

Table 1 - Nutritional composition of the food used in the experiments for the rats.

\begin{tabular}{lcc}
\hline \multicolumn{1}{c}{ Parameter } & Chow $*$ & Sunflower seeds (peeled) ** \\
\hline Humidity (\%) & 12.30 & 2.93 \\
Brute protein (\%) & 21.96 & 28.19 \\
Ether (fatty) extract (\%) & 4.61 & 58.62 \\
Mineral residue (\%) & 8.36 & 3.97 \\
Brute fiber (\%) & 4.04 & 0.36 \\
Nitrogen-free extract (\%) & 48.73 & 2.93 \\
Calcium (\%) & 1.32 & 0.22 \\
Phosphorus (\%) & 0.82 & 0.89 \\
Brute energy (Kcal/kg) & 3913 & 6888 \\
\hline
\end{tabular}

* Nuvilab CR1. Nuvital, Colombo, PR, Brazil. ** Vitao. Curitiba, PR, Brazil.

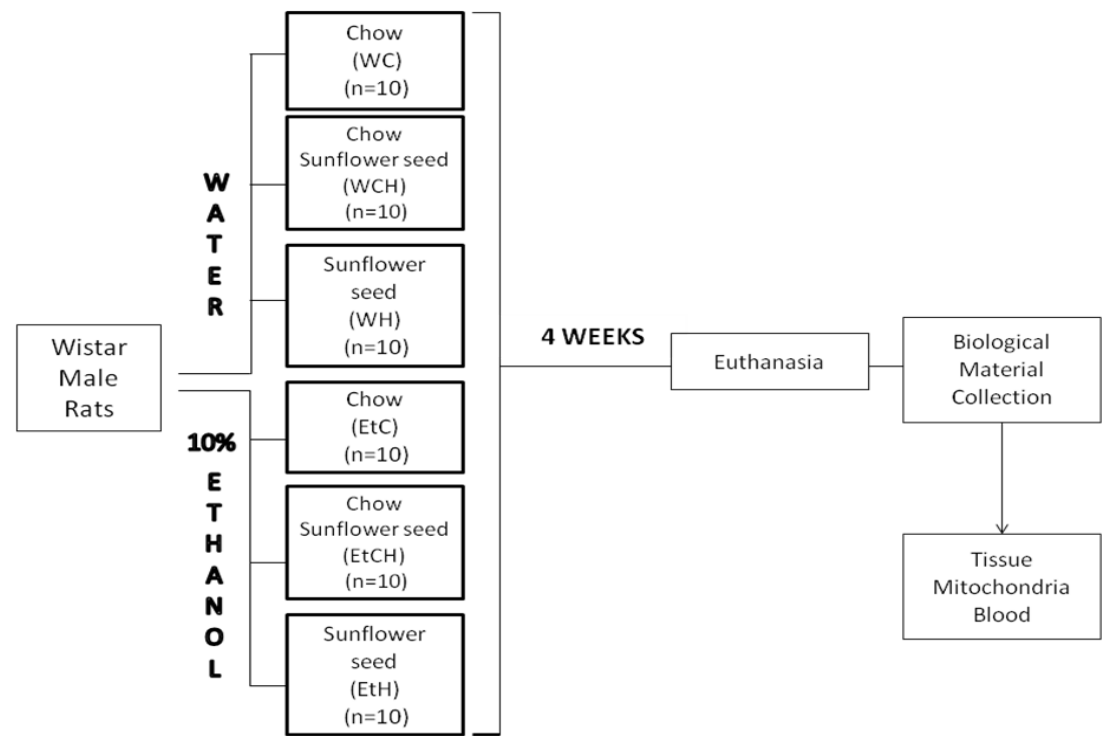

Figure 1 - Experimental designed over 4 weeks with rats fed with chow and/or sunflower seeds combined with water or $10 \%$ ethanol. Groups: WC (water-chow diet), WCH (water-chow diet and high-fat diet with sunflower seed), WH (water-high-fat diet with sunflower seed), EtC (etanol 10\%-ethanol 10\% chow diet), EtCH (ethanol 10\%-chow diet and high-fat diet with sunflower seed) and EtH (etanol 10\% high-fat diet with sunflower seed).

\section{Measurement of liver lipid content}

The lipid content in the tissue was determined by the gravimetric method (Oller do Nascimento and
Williamson 1986). All results were expressed as micrograms of triglyceride per milligram of liver tissue $\left(\mu \mathrm{g} \cdot \mathrm{mg}^{-1}\right.$ liver$)$. 


\section{Plasmatic analysis}

Plasma was obtained by centrifugation of the total blood and stored at $-80^{\circ} \mathrm{C}$. Then, cholesterol (CHO) and triglyceride (TG) levels, as well as alanine aminotransferase (ALT) and aspartate aminotransferase (AST) activity, were analyzed using a biochemical-automated system (Labtest Diagnóstica, Lagoa Santa, MG, Brazil) according to the manufacturer's instructions.

\section{Hepatic oxidative stress measurements}

Liver tissue was homogenized in phosphatebuffered saline $(\mathrm{pH} 6.5)$ using a homogenizer and centrifuged at $10,000 \times g$ at $4^{\circ} \mathrm{C}$ for $20 \mathrm{~min}$. The activities of antioxidant enzymes were determined in the supernatant. The supernatant was used to measure the activity of catalase (Cat) (Aebi 1984), superoxide dismutase (SOD) (Gao et al. 1998), and glutathione S-transferase (GST) (Habig et al. 1974). In addition, the rate of lipid peroxidation (LPO) (Jiang et al. 1991) and reduced glutathione (GSH) (Sedlak and Lindsay 1968) were measured. Results were expressed relative to the protein levels in each liver homogenate.

\section{Isolation of rat liver mitochondria}

Mitochondria were isolated from the liver by differential centrifugation as described by Voss et al. (1961) by using an extraction medium consisting of $250 \mathrm{mM}$ D-mannitol, $10 \mathrm{mM}$ HEPES (pH 7.2), $1 \mathrm{mM}$ EGTA, and $0.1 \mathrm{~g} \%$ BSA. All procedures were performed at $4{ }^{\circ} \mathrm{C}$, and the mitochondrial suspension was stored in liquid nitrogen until the activity of enzymes linked to the respiratory chain was determined.

\section{Mitochondrial enzymatic activity}

Disrupted mitochondria that were obtained by a freeze-thawing treatment were used to determine the activity of the respiratory enzyme chain. $\mathrm{NADH}$ and succinate oxidase activities were assayed polarographically (Singer and Gutman 1971). NADH dehydrogenase (EC 1.6.5.3 NADH:ubiquinone oxidoreductase) and succinate dehydrogenase (EC 1.3.5.1 succinate:ubiquinone oxidoreductase) activities were assayed spectrophotometrically according to Singer (Singer and Gutman 1971). NADH-cytochrome c reductase (EC 1.6.99.3 NADH:cytochrome c oxidoreductase) and succinate cytochrome c reductase (EC 1.3.99.1 succinate:cytochrome c oxidoreductase) activities were measured by cytochrome c reduction at $550 \mathrm{~nm}$ as described by
Somlo (1965). The activity of cytochrome c oxidase (EC 1.9.3.1 ferrocytochrome c:oxygen oxidoreductase) was determined according to Mason et al. (1973).

\section{Protein determination}

Protein concentrations in the liver homogenate for hepatic oxidative stress measurements was determined by the Bradford technique (Bradford 1976), and those from hepatic mitochondria homogenates were determined by the method described by Lowry et al. (1951). BSA was used as the standard in both methods.

\section{Statistical analysis}

The results were expressed as mean \pm standard error of the mean (SEM) and were analyzed by one-way analysis of variance (ANOVA) followed by a post-hoc Tukey-Kramer test. Two-way ANOVA was used to analyze the liquid consumption, food consumption, and body weight gain with the time (week) and the diet as the variables. The GraphPad Prism 5.0 (La Jolla, CA, USA) program was used for statistical analysis, and $\mathrm{p}<0.05$ was the value for statistical significance.

\section{RESULTS}

Diet consumption and body weight gain of rats The consumption of liquid (water or alcohol) and solid food (regular chow and/or sunflower seeds) of each animal was measured thrice per week, while the body weight was monitored once per week. The consumption of liquid over the 4-week period was the highest in animals that were fed the regular diet with water (WC), followed by the group of animals that were fed ethanol and regular chow (EtC) (Fig. 2 A). Similarly, a higher intake of solid food occurred in animals treated with the water and regular chow diet (WC), which was statistically different from all of the other groups (Fig. 2 B). With the observed differences in the amount of solid diet that was consumed, we sought to quantify the gross energy present in the diet of animals using a calorimeter $\left(\mathrm{kcal} \cdot \mathrm{kg}^{-1}\right)$ that was based on the consumption of each group. We observed that the animals that ingested the ethanol-chow and high-fat diet with sunflower seeds $(\mathrm{EtCH})$ or the ethanol-sunflower seed diet (EtH) proportionately consumed more calories than animals in the other groups (Fig. 2 C). 
However, higher calorie intake from a solid diet did not result in increasing body weight; the EtH and $\mathrm{WH}$ groups were the groups that gained the least weight over the 4-week period (Fig. 2 D).
Therefore, the combination of ethanol and sunflower seeds $(\mathrm{EtH})$ reduced the liquid and solid consumption and reduced the body weight gain, but it increased the caloric intake.
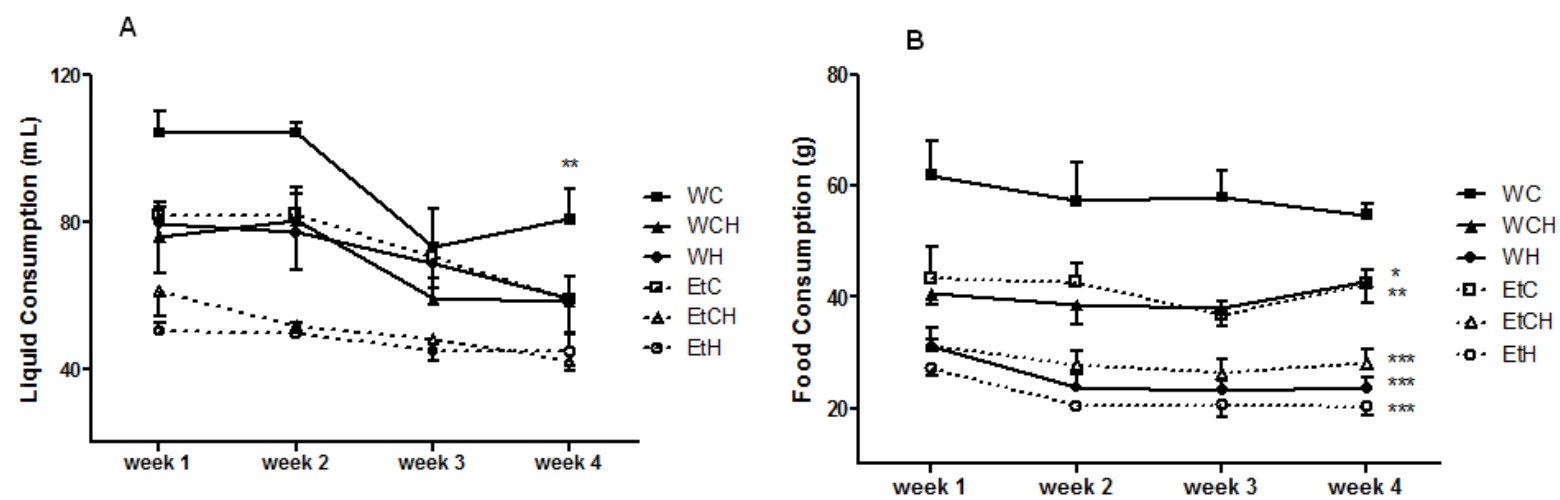

C
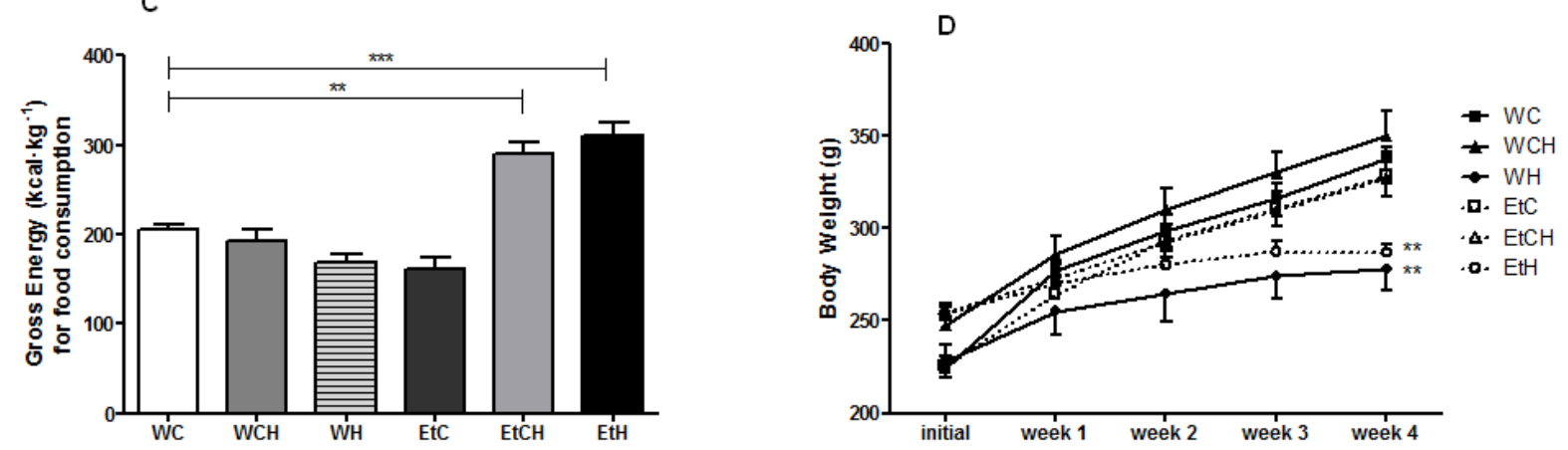

Figure 2 - Consumption of the liquids (A) and food (B) by rats fed with chow and/or sunflower seeds combined with water or $10 \%$ ethanol for 4 weeks. The gross energy ( $\mathrm{kcal} \cdot \mathrm{kg}^{-1}$ of food) derived from the consumption of the solid diet (C) and the body weight gain in rats (D) are also shown. Values are expressed as mean \pm standard error of the mean $(n=10)$, and they were analyzed by one-way analysis of variance (ANOVA) followed by the Tukey test as a post-hoc analysis for gross energy and two-way ANOVA for liquid consumption, food consumption, and body weight gain. Symbols: *, p < 0.05; **, p < 0.01 ; ***, p $<0.001$ compared to other groups (C) at the same week (A,B,D). Groups: WC (water - chow diet), WCH (water - chow diet and high-fat diet with sunflower seed), WH (water - high-fat diet with sunflower seed), EtC (10\% ethanol - chow diet), EtCH (10\% ethanol - chow diet and high-fat diet with sunflower seed) and EtH (10\% etanol - high-fat diet with sunflower seed).

The diet combination of ethanol and sunflower seeds induced severe hepatic steatosis

At macroscopic observation, the liver of the rats that were fed an HFD and ethanol (EtH and EtCH) was pale and fatty (data not shown). To confirm the lipid accumulation, histopathological analysis of the livers was performed by staining with hematoxylin-eosin. The lesions were classified according to the Rappaport score (1958). Livers of animals from the $\mathrm{WH}, \mathrm{EtCH}$, and $\mathrm{EtH}$ groups displayed predominantly microvesicular steatosis and less macrovesicular steatosis. However, no steatosis was observed in livers of animals from the EtC group after 4 weeks of the diet, and only a low degree of microsteatosis was found in the livers of animals from the WCH group. Indeed, the histology of the livers from the EtC group was similar to that of the livers from the control group (WC) (Fig. 3). Other lesions, such as lymphocytes infiltration, were also observed and quantified as shown in Table 2. The visualization of lipid droplets using Sudan Black (Fig. 3 H) staining confirmed the considerable accumulation of lipids. The hepatic lipid accumulation was detected using the gravimetric method (Fig. 4). The animals in the $\mathrm{EtH}$ and $\mathrm{EtCH}$ groups had increased intracellular lipids after 4 weeks of the diet, while the WH group presented a moderate increase that 
was not statistically significant. The EtC group presented the same level of intrahepatic lipid as the control group (WC), which indicates that the ethanol alone did not cause the accumulation of lipids in the liver. These results are consistent with the histological observations (Fig. 3).

Table 2 - Hepatic histology lesions observed in rats after receiving different diets for 4 weeks to induce steatosis.

\begin{tabular}{ccccc}
\hline Groups & Microsteatosis & Macrosteatosis & Tumefaction & Focus lymphocytosis \\
\hline WC & - & - & - & - \\
WCH & + & - & - & - \\
WH & + & + & - & + \\
EtC & - & - & - & - \\
EtCH & ++ & - & - & + \\
EtH & ++ & + & + & + \\
\hline
\end{tabular}

Symbols: -, absent; +, present; ++ highly present.

Histological score based on the Rappaport index (1958). Groups: WC (water-chow diet), WCH (water-chow diet plus high-fat diet with sunflower seeds), WH (water-high-fat diet with sunflower seeds), EtC (ethanol 10\%-chow diet), EtCH (ethanol 10\%-chow diet plus high-fat diet with sunflower seeds) and EtH (ethanol 10\%-high-fat diet with sunflower seeds).

A

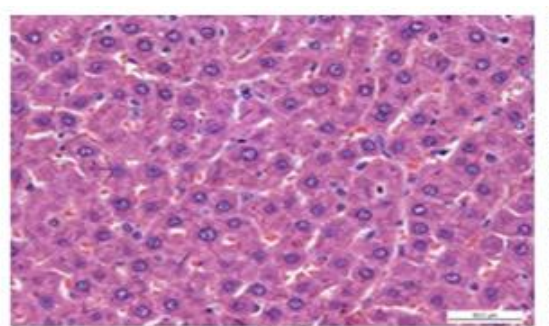

D

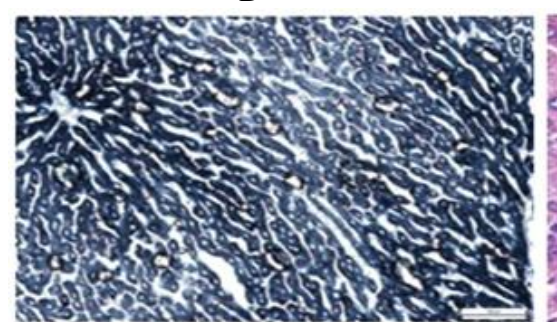

B

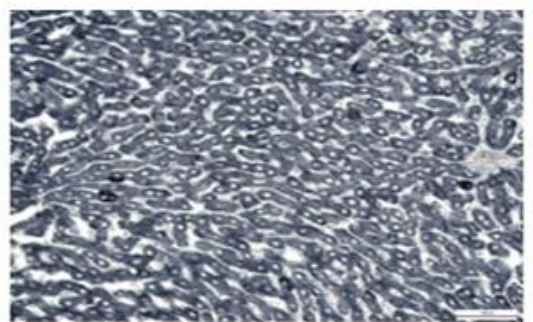

E

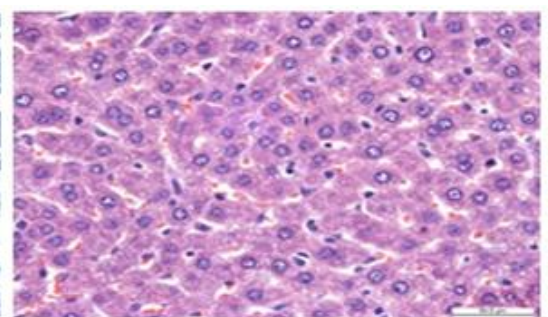

$\mathrm{H}$

G
C

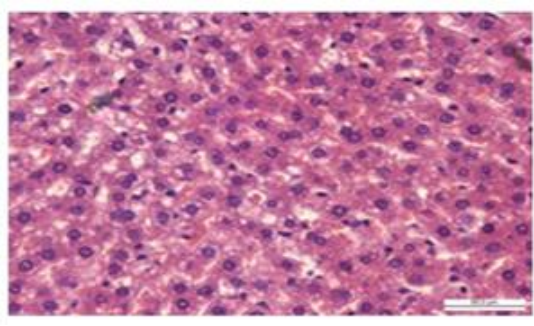

$\mathrm{F}$

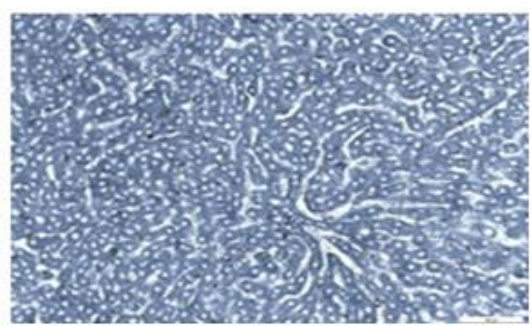

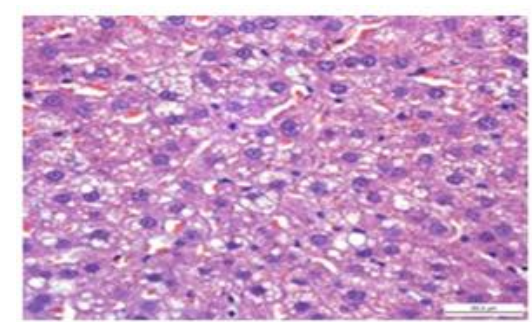

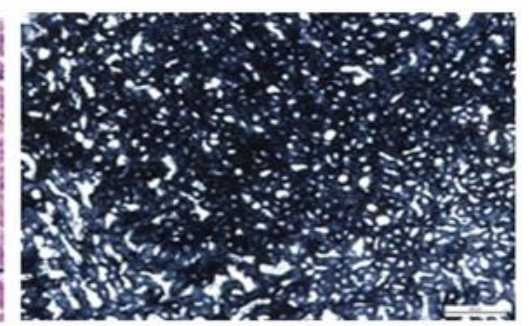

Figure 3 - Liver histology in rats after 4 weeks of the modified solid and/or liquid diet. The staining was performed with hematoxylin-eosin (A,C,E,G) and Sudan Black $(\mathrm{B}, \mathrm{D}, \mathrm{F}, \mathrm{H})$ to show the accumulation of lipids within hepatocytes. Legends: $(\mathrm{A}, \mathrm{B})$ group WC; (C,D) group WH; (E,F) group EtC; and $(\mathrm{G}, \mathrm{H})$ group EtH.

The diets influenced the plasmatic levels of ALT and cholesterol and the liver weight

The highest ALT and AST activity were observed in the groups that were fed an HFD, independent of the liquid that was consumed (water or ethanol), clearly showing the influence of the solid diet. However, the association of $10 \%$ ethanol with an
HFD increased the severity of liver damage, as illustrated by the high ALT level in the EtH group and high AST level in the WH and EtH group but not in the EtC group (Table 3). Similar results were observed for the CHO levels in the plasma: rats receiving the sunflower seed diet had increased $\mathrm{CHO}$ levels with water or ethanol. 
However, balanced diets with or without $10 \%$ ethanol influenced the triglyceride levels in rats. The relative liver weight also decreased in the animals that received only HFD or chow plus HFD (Table 3).

Table 3 - Plasmatic biochemistry and liver weight of rats fed to modified solid and liquid diet for 4 weeks to induce steatosis.

\begin{tabular}{cllllll}
\hline & WC & WCH & WH & EtC & EtCH & EtH \\
\hline ALT $\left(U \cdot L^{-1}\right)$ & $50.5 \pm 14.2$ & $35.2 \pm 10.5$ & $61.6 \pm 23,2$ & $38.9 \pm 7.0$ & $30.9 \pm 6.5$ & $62.2 \pm 18.4 *$,a \\
AST $\left(U \cdot L^{-1}\right)$ & $69.5 \pm 15.1$ & $63.9 \pm 7.8$ & $77.6 \pm 12.1^{\text {a }}$ & $59.3 \pm 5.1$ & $62.7 \pm 9.5$ & $78.7 \pm 11.5^{\text {a }}$ \\
TG $\left(m g \cdot m L^{-1}\right)$ & $86.3 \pm 11.6$ & $130.6 \pm 38.2$ & $109.7 \pm 25.8$ & $103.7 \pm 23.8$ & $163.5 \pm 31.7 *$ & $85.7 \pm 43.4$ \\
CHO $\left(m g \cdot m L^{-1}\right)$ & $52.7 \pm 22.4$ & $53.6 \pm 10.5$ & $79.1 \pm 12.2 * *, c$ & $48.7 \pm 6.6$ & $62.9 \pm 16.3$ & $90.6 \pm 16.9 * * *, \mathrm{c}$ \\
Relative liver weight $(\%)$ & $4.31 \pm 0.54$ & $3.92 \pm 0.34$ & $2.98 \pm 0.50^{* * *, c}$ & $4.29 \pm 0.22$ & $3.03 \pm 0.65 *$ & $2.70 \pm 0.32^{* * *, c}$ \\
\hline
\end{tabular}

ALT, alanine aminotransferase; AST, aspartate aminotransferase; TG, triglycerides; CHO, cholesterol. The liver weight represents the \% of the organ weight related to the body weight. Values are expressed as mean \pm standard error of the mean $(n=10)$ and were analyzed by one-way ANOVA followed by the Tukey test as a post-hoc analysis. Symbols: *, p < 0.05; **, p < 0.01; ***, p < 0.001 compared to WC; and $^{\mathrm{a}}, \mathrm{p}<0.05 ;{ }^{\mathrm{b}}, \mathrm{p}<0.01 ;{ }^{\mathrm{c}}, \mathrm{p}<0.001$ compared to EtC. Groups: WC (water-chow diet), WCH (water-chow diet plus high-fat diet with sunflower seeds), WH (water-high-fat diet with sunflower seeds), EtC (ethanol 10\%-chow diet), EtCH (ethanol 10\%-chow diet plus highfat diet with sunflower seeds) and EtH (ethanol 10\%-high-fat diet with sunflower seeds).

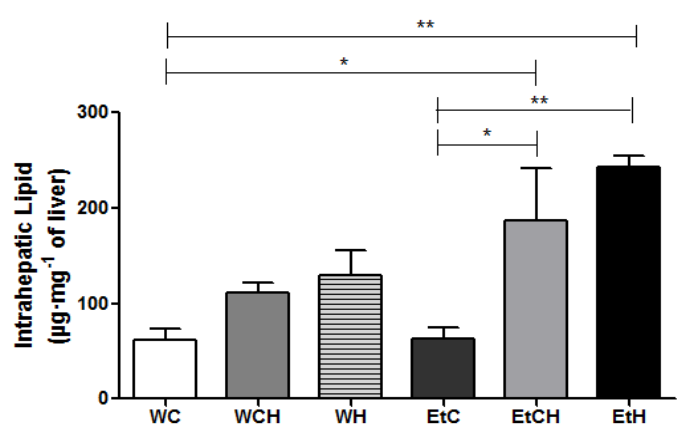

Figure 4 - Intrahepatic lipids represented by triglycerides $\left(\mu \mathrm{g} \cdot \mathrm{mg}^{-1}\right.$ liver) in rats receiving different diets. Values are expressed as mean \pm standard error of the mean $(n=10)$ and were analyzed by oneway ANOVA followed by the Tukey test as a post-hoc analysis. Symbols: *, p < 0.05; **, p < 0.01. Groups: WC (water - chow diet), WCH (water - chow diet and high-fat diet with sunflower seed), WH (water - high-fat diet with sunflower seed), EtC (10\% ethanol - chow diet), $\mathrm{EtCH}(10 \%$ ethanol - chow diet and high-fat diet with sunflower seed) and EtH (10\% etanol - high-fat diet with sunflower seed).

\section{Diet of ethanol and sunflower seeds induced hepatic oxidative stress}

The SOD activity in the EtCH and EtH groups was significantly higher $(50 \%)$ than that in the control group (WC). However, GST activity (-33\%) and GSH level $(-29 \%)$ were lower in the EtH group than in the WC group (Fig. 5 A-C). Moreover, the LPO level was higher in the $\mathrm{WH}, \mathrm{EtCH}$, and $\mathrm{EtH}$ groups, reaching about $62 \%, 112 \%$, and $137 \%$, respectively, than the level in the WC group (Fig. 5 D). These data indicate that the ethanol and HFD reduce hepatic GSH levels and GST activity and increase the SOD activity. Furthermore, LPO, despite occurring only in the presence of ethanol, was aggravated by HFD. No differences were observed in the hepatic Cat activity (data not shown).

\section{Both ethanol and diet impaired mitochondrial enzymatic activities}

The complete oxidation of NADH (Fig. 6 A) and succinate (Fig. $6 \mathrm{D}$ ) in the respiratory chain was inhibited by about $25 \%$ and $22 \%$, respectively, in the $\mathrm{WH}, \mathrm{EtC}$, and EtH groups compared to the control group (WH). We analyzed other enzymatic segments with the aim of identifying the site in the respiratory chain where the inhibition takes place. The activity of NADH dehydrogenase (complex I) was inhibited by about $13 \%, 22 \%$, and $21 \%$ in the $\mathrm{WH}, \mathrm{EtC}$, and $\mathrm{EtH}$ groups, respectively, relative to the control group (WC) (Fig. $6 \mathrm{~B}$ ). The electron transport in the segment containing NADH cytochrome c reductase was reduced by about $14 \%, 19 \%$, and $16 \%$ in the $\mathrm{WH}, \mathrm{EtC}$, and $\mathrm{EtH}$ groups, respectively (Fig. $6 \mathrm{C}$ ).

The activity of succinate dehydrogenase (complex II) was also inhibited about $23 \%, 12 \%$, and $29 \%$ in the $\mathrm{WH}, \mathrm{EtC}$, and $\mathrm{EtH}$ groups (Fig. $6 \mathrm{E}$ ), respectively. This inhibition was also observed in the segment containing succinate cytochrome $\mathrm{c}$ reductase (Fig. 6 F). No effect on cytochrome c oxidase (complex IV) was observed (data not shown). Taken together, these results show that the ethanol and/or HFD inhibited electron transport in the respiratory chain, mainly affecting complex I and II. 

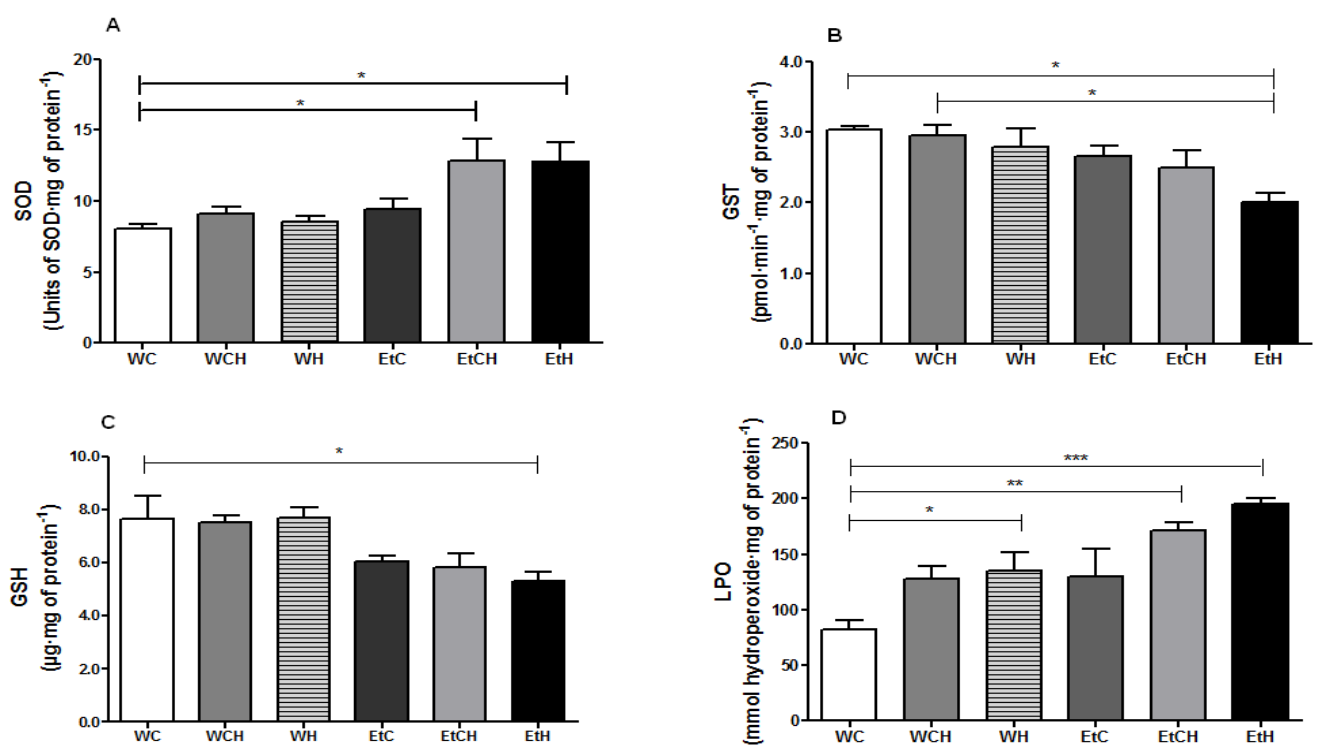

Figure 5 - Biomarkers of oxidative stress in rat liver samples collected after 4 weeks of diet. The biomarkers analyzed were superoxide dismutase (A), glutathione S-transferase (B), reduced gluthatione (C), and lipid peroxidation (D). Values represent mean \pm standard error of the mean $(n=10)$, and were analyzed by one-way ANOVA followed by the Tukey test as a post-hoc analysis. Symbols: *, p < 0.05; **, p < 0.01; ***, p < 0.001. Groups: WC (water - chow diet), WCH (water chow diet and high-fat diet with sunflower seed), WH (water - high-fat diet with sunflower seed), EtC (10\% ethanol chow diet), EtCH (10\% ethanol - chow diet and high-fat diet with sunflower seed) and EtH (10\% etanol - high-fat diet with sunflower seed).
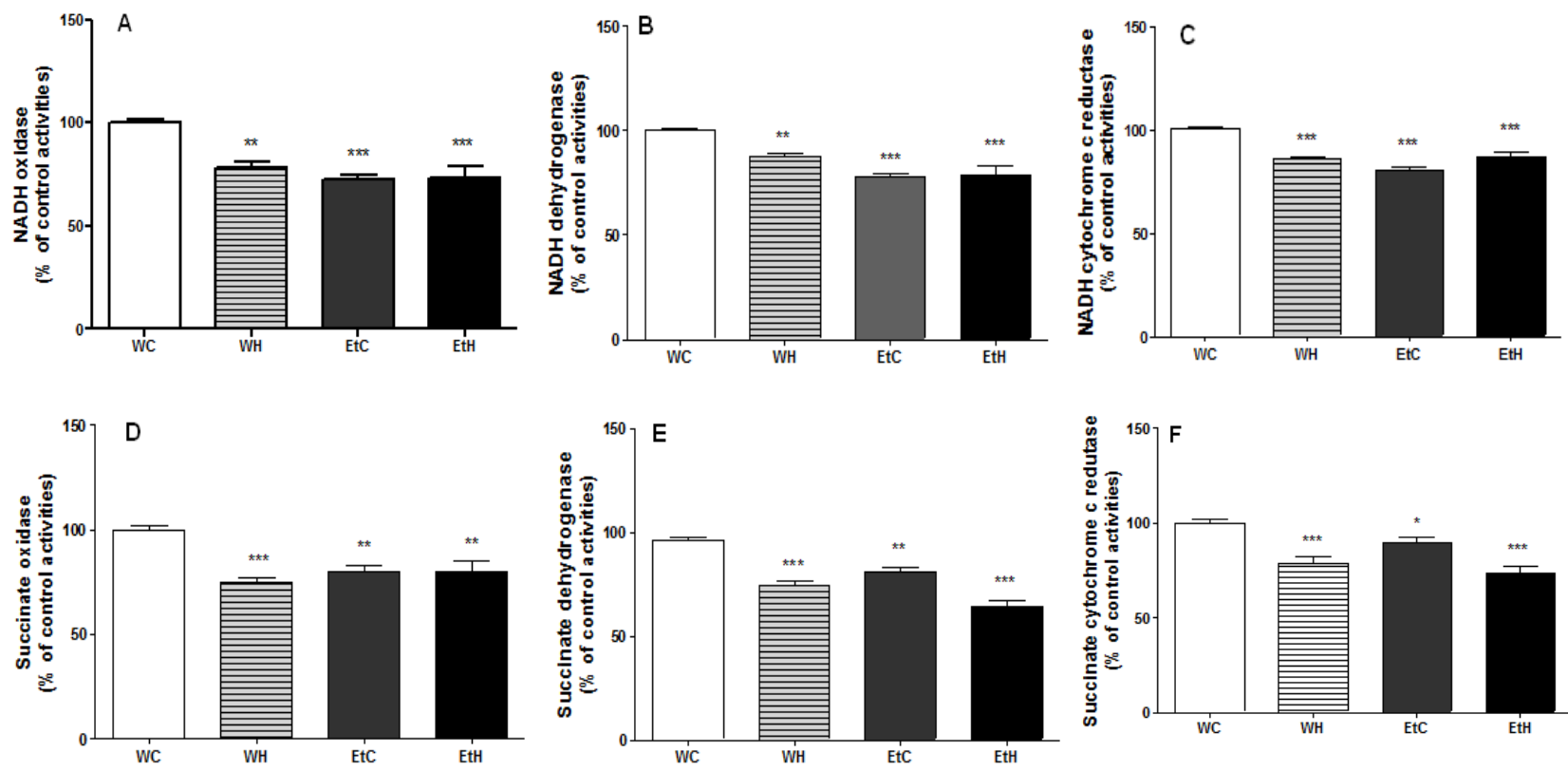

Figure 6 - Effect of high-fat diet and ethanol on the enzymatic activities of the hepatocyte respiratory chain in rats. Control values (100\%) were: (A) NADH oxidase: $73 \pm 19 \mathrm{nmol} \mathrm{O}_{2}$ consumed $\mathrm{min}^{-1} \cdot \mathrm{mg}^{-1}$ mitochondrial protein; (B) NADH dehydrogenase: $78 \pm 13 \mathrm{nmol}$ of ferricyanide reduced in $\mathrm{min}^{-1} \cdot \mathrm{mg}^{-1}$ mitochondrial protein; (C) NADH cytochrome c reductase: $86 \pm 9 \mathrm{nmol}$ of cytochrome $\mathrm{c}$ reduced $\mathrm{min}^{-1} \cdot \mathrm{g}^{-1}$ mitochondrial protein; (D) succinate oxidase: $80 \pm 18 \mathrm{nmol}$ $\mathrm{O}_{2}$ consumed $\mathrm{min}^{-1} \cdot \mathrm{mg}^{-1}$ mitochondrial protein; (E) succinate dehydrogenase: $64 \pm 13 \mathrm{nmol}$ of DCPIP reduced $\mathrm{min}^{-1} \cdot \mathrm{mg}^{-1}$ mitochondrial protein; and (F) succinate cytochrome c reductase: $73 \pm 12 \mathrm{nmol}$ of cytochrome $\mathrm{c}$ reduced $\mathrm{min}^{-1} \cdot \mathrm{mg}^{-1}$ mitochondrial protein. Results (mean \pm SEM of 4 independent experiments) are expressed as $\%$ of control activities. ANOVA followed by the Tukey test as a post-hoc analysis was used for statistical comparison. Symbols: ${ }^{*}, \mathrm{p}<0.05 ; * *, \mathrm{p}$ $<0.01 ; * * *, \mathrm{p}<0.001$. 


\section{DISCUSSION}

Hepatic steatosis is a primary response to the chronic consumption of ethanol in over $90 \%$ of individuals (Gao and Bataller 2011). In accordance with this, our data showed lipid accumulation in hepatocytes of the animals that developed AHS by consuming an HFD with $10 \%$ ethanol (Fig. 3-4). The HFD, which was represented by sunflower seeds, was selected because of the palatable taste of these seeds for rodents (Abbas and Yagoub 2008; Jabbar et al. 2008; Abdal-Gawad and Taha 2011), and because its protein amount is similar to that of the regular chow (Table 1), allowing a comparison between both diets. Another aspect that should be considered for the establishment of this model is the low cost of this diet (only 5\%) compared with the cost of a low-protein diet, which is also used in combination with ethanol in a steatosis model (Lívero 2012).

The accumulation of hepatic triglycerides is closely related to the development of liver injury. Both morphological and gravimetric methods confirmed the accumulation of intrahepatic lipids, which were mainly triglycerides (Figs. 3-4). We observed discrete microsteatosis when HFD and water were fed. However, the severity of microsteatosis was intensified when the HFD was combined with ethanol, which demonstrates the involvement of ethanol in the development of steatosis (Dey and Cederbaum 2006; Ronis et al. 2010; Bharrhan et al. 2011). In contrast, the macrosteatosis seems to be associated with HFDs, but it does not appear to be associated with ethanol (Table 2). Hepatic steatosis was present in at least $5 \%$ of histological sections, which already characterizes the liver injury (Albano 2008).

The characterization of the steatosis model investigated in this work can also be seen in the gross energy present in the food consumed by the animals over 4 weeks of receiving the diet. The rats that had a higher gross energy intake also tended to have an increased prevalence of AHS, which was confirmed in the histopathological observations (Fig. 3). It is clear that the combination of $10 \%$ ethanol and an HFD, which accelerated microsteatosis, macrosteatosis, and hepatocyte tumefaction, caused the worsening of steatosis and the deployment of the liver disease. It is important to emphasize that steatosis or other liver injuries were not induced with $10 \%$ ethanol with regular chow (EtC). The plasma levels of ALT and AST, which is an important indicator of cellular hepatic lesions (Sathaye et al. 2011; Chacko et al. 2011), were increased in the $\mathrm{WH}$ and EtH groups, but it was not in EtC group (Table 3). ALT is very active in the liver; thus, it can be easily detected in small quantities in the plasma after liver injuries. Enzyme release can be caused by hepatocyte lysis and by increases in plasma membrane permeability (Babcock et al. 1981; De Oliveira Christoff et al. 2008). Our data, which suggest that the plasma ALT is more influenced by the HFD than by the ethanol, corroborate those of Demori et al. (2006).

Hochgraf et al. (Hochgraf et al. 1997) demonstrated that oxidized linoleic acid, which is present in sunflower seeds, promotes a significant increase in the plasma cholesterol levels in rats. In contrast, some other studies reported reduced levels of plasma cholesterol and triglycerides in rats and pigs in response to the consumption of oxidized dietary oil (Eder and Kirchgessner 1999; Eder 1999; Eder and Stangl 2000; Eder et al. 2003; Acikgoz et al. 2011). In this context, our results demonstrated increased cholesterol levels with HFD, which agrees with the proposed of Hochgraf et al. (Hochgraf et al. 1997). This increase was not associated or intensified by the presence of ethanol in the diet (Table 3). The higher plasma cholesterol levels might be related to impaired liver uptake of cholesterol (Hochgraf et al. 1997). Considering the liver injuries observed, we measured biomarkers of hepatic oxidative stress, because increases of oxidative stress are an essential factor in the development of secondary lesions of chronic alcoholism (Henzel et al. 2004). Chronic ethanol consumption diminishes the level of cellular antioxidants such as reduced glutathione and renders hepatocytes more susceptible to free radical-induced injury by means of unimpeded lipid peroxidation. Our results corroborate this idea because the hepatic $\mathrm{GSH}$ level was reduced in the $\mathrm{EtH}, \mathrm{EtC}$, and $\mathrm{EtCH}$ groups (Fig. 5 C). According to these findings, the activity of GST, which is involved in the metabolism of xenobiotics and also has an important antioxidant function, was decreased in the EtH and EtCH groups (Fig. 5 B), showing a partial reduction in the detoxification capacity of those livers ( $\mathrm{Lu}$ and Cederbaum 2008; Tiwari and Chopra 2012). Also, the enzymatic activity of SOD increased in the presence of ethanol and the HFD (Fig. 5 A). SOD is highly efficient in the catalytic removal of $\mathrm{O}_{2}{ }^{--}$through its dismutation to $\mathrm{H}_{2} \mathrm{O}_{2}$ (Tiwari and Chopra 2012). Increased SOD 
activity can lead to the production of toxic levels of $\mathrm{H}_{2} \mathrm{O}_{2}$ because it is generated from ${ }^{\circ} \mathrm{OH}$, which is more reactive than $\mathrm{O}_{2}{ }^{\circ}$. The increased ${ }^{\circ} \mathrm{OH}$ levels could be prevented by Cat, which reduces $\mathrm{H}_{2} \mathrm{O}_{2}$ to water. Thus, simultaneous to SOD role, a rise in Cat activity is essential for an overall beneficial effect and an increased SOD activity (Sathaye et al. 2011). In our study, the higher SOD activity was not accompanied by changes in Cat activity, which could promote the accumulation of $\mathrm{H}_{2} \mathrm{O}_{2}$ and, consequently, an increase in the generation of $\cdot \mathrm{OH}$. This radical can attack hepatocyte membranes, resulting in a significant lipoperoxidation. The increased level of LPO that we observed in the livers from both the EtH and $\mathrm{EtCH}$ groups confirmed this hypothesis (Fig. 5 D). A larger attack on free fatty acids in the membrane of hepatocytes reduces mitochondrial activity (Yang et al. 2000), as observed in our study. Fat accumulation in hepatocytes is the result of imbalanced fat metabolism, such as decreased mitochondrial lipid oxidation and enhanced synthesis of triglycerides. Therefore, the development of hepatic steatosis is associated with increased values of oxidative stress and structural defects in mitochondria (Sanyal et al. 2001; Carabelli et al. 2011), and it impacts mitochondrial respiration (Carabelli et al. 2011). In this work, the activity of segments of the mitochondrial respiratory chain was analyzed in isolated hepatic mitochondria, thus accessing the sites in the respiratory chain on which inhibitions in response to diet occur. Our results show that the oxidation of NADH and succinate was partially inhibited in the $\mathrm{WH}, \mathrm{EtCH}$, and $\mathrm{EtH}$ groups. Since no difference among the groups was observed, we suggest that the presence of ethanol in the diet was the main determinant of the inhibition. This result is consistent with that of Chacko et al. (Chacko et al. 2011). Also, the combination of dietary ethanol and HFD seems to increase this inhibition. In fact, the activities of complexes I and II were impaired, and complex I was the most affected. These results suggest that the combination of ethanol and sunflower seeds in the diet impairs the mitochondria in AHS, contributing to decreased functioning of the oxidative phosphorylation system and depressed rates of ATP synthesis.

Despite of the forced ethanol consumption in drinking water of the rats, what can be pointed as a limitation of the proposal method (BrandonWarner et al. 2012), this model was efficient for investigating the pathological aspects of AHS.
Liver steatosis was successfully induced in rats after 4 weeks of receiving a diet with $10 \%$ ethanol and HFD. The liver function was modified and the alterations were identified by morphological analysis, oxidative stress biomarkers, plasmatic parameters, and mitochondrial activity. Thus, the combination of ethanol and sunflower seeds produced an interesting and inexpensive model to study ALD that can be used as a pathological or pharmacological tool in this field of investigation.

\section{ACKNOWLEDGEMENTS}

The authors are grateful to Isabella Aviles Fabosi for the help in all experiments during this work, Dr. Lauro Mera de Souza (UFPR) for the inestimable help in the gravimetry analysis, and to Fundação Araucária and CAPES for the financial support.

\section{REFERENCES}

Abbas TEE, Yagoub YM. Sunflower cake as a substitute for groundnut cake in commercial broiler chick diets. Pakistan J Nut. 2008; 7: 782-784.

Abdal-Gawad H, Taha H. Bioavailability and toxicological potential of sunflower-bound residues of 14 C-chlorpyrifos insecticide in rats. J Environ Sci Health. Part B. 2011; 46: 683-690.

Acikgoz Z, Bayraktar H, Altan O, Akhisaroglu ST, Kirkpınar F, Altun Z. The effects of moderately oxidised dietary oil with or without vitamin E supplementation on performance, nutrient digestibility, some blood traits, lipid peroxidation and antioxidant defence of male broilers. J Sci Food Agric. 2011; 91: 1277-1282.

Aebi H. Catalase in vitro. Methods Enzymol. 1984; 105: 121-126.

Albano E. New concepts in the pathogenesis of alcoholic liver disease. Expert Rev Gastroenterol Hepatol. 2008; 6: 749-759.

Albano E. Oxidative mechanisms in the pathogenesis of alcoholic liver disease: Review. Mol Aspects Med. 2008; 29: 9-16.

Babcock JL, Suber RL, Frith CH, Geren CR. Systemic effect in mice of venom apparatus extract and toxin from the brown recluse spider (Loxosceles reclusa). Toxicon. 1981; 19: 463-471.

Bharrhan S, Koul A, Chopra K, Rishi P. Catechin suppresses an array of signaling molecules and modulates alcohol-induced endotoxin mediated liver injury in a rat model. PLoS One. 2011; 6: e20635. 
Bradford MM. A rapid and sensitive method for the quantitation of microgram quantities of protein utilizing the principle of protein dye binding. USA Anal Biochem. 1976; 72: 248-254.

Brandon-Warner E, Schrum LW, Schmidt CM, McKillop IH. Rodent models of alcoholic liver disease: of mice and men. Alcohol. 2012; 46: 715725.

Byun JS, Jeong WI. Involvement of hepatic innate immunity in alcoholic liver disease. Immune Netw. 2010; 10: 181-187.

Caballero VJ, Mendieta JR, Giudici AM, Crupkin AC, Barbeito CG, Ronchi VP, et al. Alternation between dietary protein depletion and normal feeding cause liver damage in mouse. J Physiol Biochem. 2011; 67: 43-52.

Carabelli J, Burgueño AL, Rosselli MS, Gianotti TF, Lago NR, Pirola CJ, et al. High fat diet-induced liver steatosis promotes an increase in liver mitochondrial biogenesis in response to hypoxia. $J$ Cell Mol Med. 2011: 15: 1329-1338.

Chacko BK, Srivastava A, Johnson MS, Benavides GA, Chang MJ, Jhala N, et al. Mitochondria-targeted ubiquinone (MitoQ) decreases ethanol-dependent micro and macro hepatosteatosis. Hepatology. 2011; 54: 153-163.

Comporti M, Signorini C, Leoncini S, Gardi C, Ciccoli L, Giardini A, et al. Ethanol-induced oxidative stress: basic knowledge. Genes Nutr. 2010; 5: 101-109.

Curry-McCoy TV, Osna NA, Nanji AA, Donohue TMJr. Chronic ethanol consumption results in atypical liver injury in copper/zinc superoxide dismutase deficient mice. Alcohol Clin Exp Res. 2010; 34: 251-261.

de Oliveira Christoff A, de Oliveira A, Chaim OM, Lugarini D, Bastos Pereira AL, Paludo KS, et al, Effects of the venom and the dermonecrotic toxin LiRecDT1 of Loxosceles intermedia in the rat liver. Toxicon. 2008; 52: 695-704.

Demori I, Voci A, Fugassa E, Burlando B. Combined effects of high-fat diet and ethanol induce oxidative stress in rat liver. Alcohol. 2006; 40: 185-191.

Dey A, Cederbaum AI. Alcohol and oxidative liver injury. Hepatology. 2006; 43: S63-74.

Di Pascoli L, Lion A, Milazzo D, Caregaro L. Acute liver damage in anorexia nervosa. Int $J$ Eat Disord. 2004; 36: 114-117.

Eder K, Kirchgessner M. The effect of a moderately thermoxidised dietary fat on the vitamin E status, the fatty acid composition of tissue lipids, and the susceptibility of low-density lipoproteins to lipid peroxidation in rats. Lipids. 1999; 101: 178-184.

Eder K, Stangl GI. Plasma thyroxine and cholesterol concentrations of miniature pigs are influenced by thermally oxidised dietary lipids. J Nutr. 2000; 130: 116-121.
Eder K, Keller U, Hirche F, Brandsch C. Thermally oxidised dietary fats increase the susceptibility of rat LDL to lipid peroxidation but not their uptake by macrophages. J Nutr. 2003; 133: 2830-2837.

Eder K. The effects of a dietary oxidised oil on lipid metabolism in rats. Lipids. 1999; 34: 717-725.

Gao B, Bataller R. Alcoholic Liver Disease: Pathogenesis and New Therapeutic Targets. Gastroenterology. 2011; 141: 1572-1585.

Gao R, Yuan Z, Zhao Z, Gao X. Mechanism of pyrogallol autoxidation and determination of superoxide dismutase enzyme activity. Bioelectrochem Bioenerg. 1998; 45: 41-45.

Habig WH, Papst MJ, Jakoby WB. Glutathione Stransferases: the first enzymatic step in mercapturic acid formation. J Biol Chem. 1974; 249: 7130-7139.

Henzel K, Thorborg C, Hofmann M, Zimmer G, Leuschner U. Toxicity of ethanol and acetaldehyde in hepatocytes treated with ursodeoxycholic or tauroursodeoxycholic acid. Biochim Biophys Acta. 2004; 1644: 37-45.

Hochgraf E, Mokady S, Cogan U. Dietary oxidised linoleic acid modifies lipid composition of rat liver microsomes and increases their fluidity. J Nutr. 1997; 127: 681-686.

Jabbar MA, Ahmad S, Riffat S. Effect of replacing cotton seed cake with sunflower meal in the rations of lactating crossbred cows. J Vet Anim Sci. 2008; 1: 1113.

Jiang ZY, Woollard AC, Wolff SP. Lipid hydroperoxide measurement by oxidation of $\mathrm{Fe}_{2}^{+}$in the presence of xylenol orange. Comparison with the TBA assay and an iodometric method. Lipids. 1991; 26: 853-856.

Korourian S, Hakkak R, Ronis MJ, Shelnutt SR, Waldron J, Ingelman-Sundberg et al . Diet and risk of ethanol-induced hepatotoxicity: carbohydratefat relationships in rats. Toxicol Sci. 1999; 47: 110117.

Liber CS. Alcoholic fatty liver: its pathogenesis and mechanism of progression to inflammation and fibrosis. Alcohol. 2004; 34: 9-19.

Lívero FAR. Esteatose hepática alcoólica: correlações com receptor nuclear FXR e estresse oxidativo (FXR agonist 6ECDCA reduces hepatic steatosis and oxidative stress induced by ethanol in mice). Master Thesis 2012. Federal University of Paraná, Curitiba, Brazil, http://hdl.handle.net/1884/27139.

Lowry OH, Rosebrough NJ, Farr AL, Randall RJ. Protein measurement whit the Folin phenol reagent. $J$ Biol Chem. 1951; 193: 265-275.

Lu Y, Cederbaum AI. CYP2E1 and oxidative liver injury by alcohol. Free Radic Biol Med. 2008; 44: 723-38. 
Mason TL, Poyton RO, Wharton DC, Schatz G. Cytochrome c oxidase from bakers yeast, isolation and properties. J Biol Chem. 1973; 248: 1346-1354.

Miranda-Mendez A, Lugo-Baruqui A, ArmendarizBorunda J. Molecular basis and current treatment for alcoholic liver disease. Int J Environ Res Public Health. 2010; 7: 1872-1888.

Oller do Nascimento CM, Williamson DH. Evidence for conservation of dietary lipid in the rat during lactation and the immediate period after removal of the litter. Decreased oxidation of oral [1-14C] triolein. Biochem J. 1986; 239: 233-236.

Pessayre D, Fromenty B, Berson A, Robin MA, Lettéron P, Moreau R, et al. Central role of mitochondria in drug-induced liver injury. Drug Metab Rev. 2012; 44: 34-87.

Pessayre D, Fromenty B. NASH a mitochondrial disease. J Hepatol. 2005; 42: 928-940.

Powel EE, Jonsson JR, Clouston AD. Steatosis: cofactor in other liver diseases. Hepatology. 2005; 42: 5-13.

Rautou PE, Cazals-Hatem D, Moreau R, Francoz C, Feldmann G, Lebrec D, et al. Acute liver cell damage in patients with anorexia nervosa: a possible role of starvation-induced hepatocyte autophagy. Gastroenterology. 2008; 135: 840-848.

Ronis MJ, Korourian S, Blackburn ML, Badeaux J, Badger TM. The role of ethanol metabolism in development of alcoholic steatohepatitis in the rat. Alcohol. 2010; 44: 157-169.

Sanyal AJ, Campbell-Sargent C, Mirshahi F, Rizzo WB, Contos MJ, Sterling RK, et al. Nonalcoholic steatohepatitis: association of insulin resistance and mitochondrial abnormalities. Gastroenterology. 2001; 120: 1183-1192.
Sathaye S, Bagul Y, Gupta S, Kaur H, Redkar R. Hepatoprotective effects of aqueous leaf extract and crude isolates of Murraya koenigii against in vitro ethanol-induced hepatotoxicity model. Exp Toxicol Pathol. 2011; 63: 587-591.

Sedlak J, Lindsay RH. Estimation of total, proteinbound, and nonprotein sulfhydryl groups in tissue with Ellman's reagent. Anal Biochem. 1968; 25: 192205.

Singer TP, Gutman M. The DPNH dehydrogenase of the mitochondrial respiratory chain. Adv Enzymol Relat Areas Mol Biol. 1971; 34: 79-153.

Somlo M. Induction des lactico-cytocrome c reductases (D-ET L-) de la levure aerobie par les lactates (D-ETL). Biochim Biophys Acta. 1965; 97: 183-201.

Tilg H, Moschen AR, Kaneider NC. Pathways of liver injury in alcoholic liver disease. J Hepatol. 2011; 55: 1159-1161.

Tiwari V, Chopra K. Attenuation of oxidative stress, neuroinflammation, and apoptosis by curcumin prevents cognitive deficits in rats postnatally exposed to ethanol. Psychopharmacology. 2012; 224: 519535.

Yang S, Zhu H, Li Y, Lin H, Gabrielson K, Trush MA, et al. Mitochondrial adaptations to obesityrelated oxidant stress. Arch Biochem Biophys. 2000; 378: 259-268. 